\title{
Comparison of the diagnostic accuracy of four smartphone-compatible blood pressure monitors in post-myocardial infarction patients
}

Journal of Telemedicine and Telecare $0(0)$ I-6

(C) The Author(s) 2017

Reprints and permissions: sagepub.co.uk/journalsPermissions.nav DOI: I0.II77/I357633XI7704092 journals.sagepub.com/home/jtt

@SAGE

\author{
Roderick W Treskes', Ron Wolterbeek ${ }^{2}$, Enno T van der Velde', \\ Daniëlle C Eindhoven' and Martin J Schalij'
}

\begin{abstract}
Introduction: Smartphone-compatible blood pressure devices may be a good alternative to enable self-measurement of blood pressure by patients. Furthermore, automatic transferral of data to the hospital allows for remote monitoring. To our knowledge, no study has compared four of these smartphone-compatible blood pressure devices.

Methods: Patients who were followed up for acute myocardial infarction were asked to participate during their outpatient clinic visit. After five minutes of rest, six blood pressure devices were applied. The order was randomised. Four devices were smartphone-compatible. One device was an automated oscillometric device. One device was a handheld aneroid sphygmomanometer (reference device). All measurements were compared using a linear mixed model.

Results: A total of 43 patients (62.7 \pm II.3 years, $79 \%$ male) were included. Compared to the reference device, four blood pressure monitors yielded a significant higher mean systolic blood pressure and four monitors yielded a significant higher diastolic BP. One device yielded a non-significant lower mean systolic blood pressure and one device yielded a non-significant higher mean diastolic blood pressure. Except for one blood pressure device, all mean differences were smaller than $5 \mathrm{mmHg}$. Conclusion: In this study, average inter-device variability was shown to be statistically significant, however four devices remained within the predefined range of $5 \mathrm{mmHg}$ for both systolic and diastolic blood pressures.
\end{abstract}

\section{Keywords}

Ehealth, home telecare, telecardiology, self care

Date received: 10 February 2017; Date accepted: 9 March 2017

\section{Introduction}

Blood pressure (BP) measurement is inevitable in BP control, which is considered a cornerstone in the primary and secondary prevention of cardiovascular disease. ${ }^{1} \mathrm{BP}$ measurement is classically done by a trained healthcare professional using a mercury sphygmomanometer and a stethoscope to generate and auscultate the Korotkoff sounds. ${ }^{2}$ Although this method is considered the gold standard, it has some disadvantages: first, involvement of a physician brings in the so-called 'white coat hypertension' phenomenon, in which the BP significantly rises in the presence of a physician. ${ }^{3}$ Due to this phenomenon, the office BP measurement could inadequately represent the patient's BP. ${ }^{3}$ Second, patients often have to come to the outpatient clinic, making the measurement timeconsuming and a burden to the patient. Lastly, due to the necessity of the presence of a healthcare professional and an outpatient clinic, it is costs more than home BP measurement. 4
An alternative to outpatient clinic BP measurement is home BP measurement, in which patients use an automated device to measure their BP at home without the presence of trained healthcare staff. Home BP measurement has advantages, as patients do not have to go to the outpatient clinic and it bypasses the white coat hypertension phenomenon. ${ }^{5}$ For these reasons, the European Hypertension Society guidelines stress the importance of the use of home BP measurement noting that, using home BP measuring, cardiovascular morbidity and mortality

\footnotetext{
'Department of Cardiology, Leiden University Medical Center,

The Netherlands

${ }^{2}$ Department of Medical Statistics, Leiden University Medical Center,

The Netherlands
}

\section{Corresponding author:}

Roderick W Treskes, Department of Cardiology, Leiden University Medical Center, PO Box 9600, 2300 RC Leiden, The Netherlands.

Email: r.w.treskes@lumc.nl 
can be predicted significantly better than with office measurements. ${ }^{5}$

Furthermore, it was recently demonstrated that home BP monitoring with antihypertensive treatment adjustments by pharmacists even improved BP control compared to usual care. ${ }^{6}$

Over the past few years, smartphone-compatible oscillometric BP monitors have been approved for overthe-counter sale in the European Union. ${ }^{7-9}$ These BP monitors can be applied by the patient without the presence of trained healthcare professionals. Furthermore, the storage of data in the Cloud allows for easy and automatic transferral to healthcare professionals. ${ }^{7-9}$ Thus, these smartphone-compatible BP monitors could be used for more frequent measurements, without the presence or assistance of a trained healthcare professional and the necessity of an outpatient clinic. These features make these smartphone-compatible BP monitors suitable for telemedicine. So far, most studies have focused on the validation of one smartphone-compatible device in healthy volunteers. ${ }^{10-13}$ To our knowledge, no study has yet compared the performances of these four smartphonecompatible monitors in a population with a doctorconfirmed cardiovascular disease. Therefore, the aim of this study is to compare the intra- and inter-device variability of four smartphone compatible BP monitors with an aneroid sphygmomanometer and an automated, nonsmartphone compatible, oscillometric monitor in a population with recent acute myocardial infarction (AMI).

\section{Methods}

\section{Patient population}

Patients with recent ( $<1$ year) AMI visiting the outpatient clinic as part of the follow-up of their AMI were approached to participate in the study. Patients were excluded if they had had a documented history of an irregular heart rhythm (e.g. atrial fibrillation, atrial flutter, Mobitz II or grade III atrioventricular-block). Furthermore, patients were excluded if they were considered an incapacitated adult, if they were pregnant or if they were unwilling to sign the informed consent form. Patients who did not own a smartphone were not excluded for this study.

\section{BP monitors}

Details on the methods of this study and the BP monitors used have previously been described in a conference paper. ${ }^{14}$ For this study, six BP monitors were used. The Welch Allyn 767 (Welch Allyn, Skaneateles Falls, New York, USA), the Omron M7 (Omron, Kyoto, Japan), the Withings Wireless Blood Pressure Monitor (Withings, Issy-les-Moulineaux, France), iHealth BP5 (iHealth Lab, Inc., Mountain View, California, USA), QardioArm (Qardio Inc., San Francisco, California, USA) and the iHealth BP7 (iHealth Lab, Inc.,
Mountain View, California, USA). All devices bear a Conformité Européenne (CE) mark and are approved by the Food and Drug Administration (FDA). The Withings Wireless Blood Pressure Monitor, QardioArm, iHealth BP5 and iHealth BP7 are all automated oscillometric devices that are smartphone-compatible. These four BP monitors communicate with the smartphone via Bluetooth. Inflation and deflation is automated and started by a command from the smartphone. Results of measurements are sent to the device's dedicated smartphone application (app). The Withings Wireless Blood Pressure Monitor, the QardioArm and the iHealth BP5 are placed around the bare upper arm of the patient. The iHealth BP7 is placed around the bare wrist of the patient. The Omron M7 is an automated oscillometric device. It is placed around the bare upper arm of the patient. Inflation and deflation are automated and started by pushing a button on the device. The Omron M7 is not smartphone compatible.

The Welch Allyn 767 is a handheld aneroid sphygmomanometer. It is placed around the bare upper arm of the patient. The Welch Allyn 767 has to be applied by a trained physician. The device has to be pumped up until the pressure is $10 \mathrm{mmHg}$ higher than the systolic $\mathrm{BP}$ of the patients. While deflating, a trained physician needs to auscultate the Korotkoff sounds at the elbow joint. Upon hearing the first Korotkoff sounds, the systolic BP can be determined. When the Korotkoff sounds disappear, the diastolic BP can be determined. ${ }^{15}$

\section{Study procedures}

Patients were taken to a separate, quiet room. They were asked to sit down and relax for at least five minutes. During these five minutes, the order of applying the devices was randomised. For randomisation, a website (www.randomizer.org) was used. After five minutes of rest, the devices were, one-by-one, applied to the patient. To assure that the devices were applied according to the device manufacturer's instruction manual, a projectdedicated healthcare professional applied the device. The arm of patient's preference was used. All devices were applied to the same arm of the patient. Patients were not allowed to talk or drink during the measurements.

The Omron M7, the Withings Wireless Blood Pressure Monitor, the iHealth BP5, the QardioArm and the iHealth BP7 were applied by one of the investigators (RWT). The Welch Allyn 767 was applied by an independent trained physician (DCE), who was blinded to the outcomes of the other measurements. The patient was blinded to all results of all measurements. After the last device last measurement, results of all measurements were discussed with the patient. After a device was applied, it was inflated and deflated three times consecutively, generating three systolic BPs and three diastolic BPs. The average of these three systolic BP measurements was taken as systolic and the average of these three diastolic BP measurements was taken as diastolic BP. 


\section{Statistical analysis}

IBM SPSS Statistics for Windows, version 23 (IBM Corp., Armonk, New York, USA) was used for statistical analysis. Continuous variables were presented as mean \pm standard deviation or as medians with interquartile range (IQR). The analyses were based on the repeated measures design with correlated BPs within patients. Linear mixed models were applied to accommodate this design to compare mean BPs and the variation (variances) of measurements among devices. A $p$-value of less than 0.05 was considered statistically significant. The variances of the measurements within devices were compared within linear mixed models by likelihood ratio tests comparing models with equal variances versus models with unequal variances within an appropriate chi-square distribution.

\section{Sample size calculation}

The power calculation was done in PASS (PASS 15. NCSS, LLC. Kaysville, Utah, USA, ncss.com/software/ pass) and was based on paired $t$-tests of smartphone device measurements against the measurements of the standard device, assuming a clinically relevant difference of $5 \mathrm{mmHg}$, a standard deviation of the measurements of 11.4 and a within-patient Pearson's correlation of 0.8 . The calculated sample size was 43 patients.

\section{Ethical conduct}

This study was done in accordance with the Declaration of Helsinki and Good Clinical Practice Guidelines. The study was approved by the local Research and Ethical Board. All study participants provided written informed consent before participation. All devices for this study were bought from the manufacturers. No manufacturer had a role in the study design, conduction of the study, data analysis or the decision to submit the article for publication.

\section{Results}

\section{Study population}

A total of 43 study participants ( $79 \%$ male) were included in the study. Mean age was $62.7 \pm 11.3$ (range 39.5-81.6). Median body mass index (BMI) was 26.6 (interquartile range (IQR) 24.2-29.3). The mean systolic BP (as measured by the Welch Allyn 767) was $119 \pm 14$ (minimum-maximum range 97-160). The mean diastolic BP was $71 \pm 8$ (minimum-maximum range 50-90). All measurements yielded 258 systolic BPs and 258 diastolic BPs (Figure 1).

\section{Comparison of means}

The average systolic BP measured by Welch Allyn was 118.9. The average systolic $\mathrm{BP}$ was $+0.25(95 \%$ confidence interval (CI) $(-2.9-2.4), p=0.856)$ as measured by Omron,

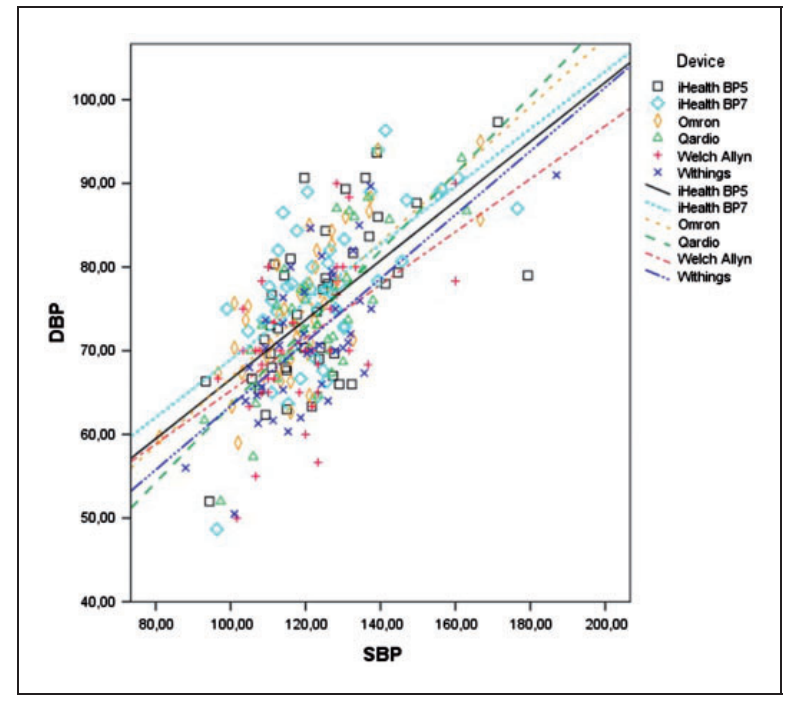

Figure I. Scatterplot of the 258 measurements by iHealth BP5, iHealth BP7, Omron M7, Qardio, Welch Allyn and Withings. The $x$-axis displays the systolic blood pressure (SBP). The $y$-axis displays the diastolic blood pressure (DBP). The lines displayed are the regression lines per device.

Table I. Results of systolic blood pressure (BP) measurements of all oscillometric devices, compared with the Welch Allyn.

\begin{tabular}{lllll}
\hline & $\begin{array}{l}\text { Mean } \\
\text { systolic } \\
\text { Device }\end{array}$ & $\begin{array}{l}\text { Difference } \\
\text { with Welch } \\
\text { Allyn }\end{array}$ & $\begin{array}{l}\text { 95\% } \\
\text { Confidence } \\
\text { interval }\end{array}$ & $p$-Value \\
\hline Omron & I 18.6 & -0.25 & $(-2.9-2.4)$ & $p=0.856$ \\
iHealth BP5 & 124.0 & +5.0 & $(2.3-7.7)$ & $p<0.001$ \\
iHealth BP7 & 126.0 & +7.1 & $(4.4-9.7)$ & $p<0.001$ \\
Qardio & 123.0 & +4.1 & $(1.3-6.8)$ & $p=0.004$ \\
Withings & I22.1 & +3.2 & $(0.5-5.9)$ & $p=0.022$ \\
\hline
\end{tabular}

was $+5.0(95 \%$ CI $(2.3-7.7), p<0.001)$ as measured by iHealth BP5, was +7.1 (95\% CI $(4.4-9.7), p<0.001)$ as measured by iHealth BP7, was +4.1 (95\% CI $(1.3-6.8)$, $p=0.004)$ as measured by Qardio and was $+3.2(95 \%$ CI $(0.5-5.9), p=0.022)$ as measured by Withings. These results are summarised in Table 1.

The average diastolic BP measured by Welch Allyn was 71.2. The average diastolic BP was $+3.2(95 \% \mathrm{CI}$ $(1.7-4.5), p<0.001)$ as measured by Omron, was +3.9 (95\% CI $(2.5-5.3), p<0.001)$ as measured by iHealth BP5, was $+6.7(95 \%$ CI $(5.3-8.1), p<0.001)$ as measured by iHealth BP7, was $+2.9(95 \%$ CI $(1.5-4.4), p<0.001)$ as measured by Qardio and was +0.7 (95\% CI (-0.7-2.2), $p=0.324)$ as measured by Withings. These results are summarised in Table 2.

\section{Comparison of means of smartphone compatible monitors}

The average systolic BP measured by Withings was 122.1 . Differences with iHealth BP5 and Qardio were not 
Table 2. Results of diastolic blood pressure (BP) measurements of all oscillometric devices, compared with the Welch Allyn.

\begin{tabular}{lllll}
\hline & $\begin{array}{l}\text { Mean } \\
\text { diastolic } \\
\text { BP }\end{array}$ & $\begin{array}{l}\text { Difference } \\
\text { with Welch } \\
\text { Allyn }\end{array}$ & $\begin{array}{l}\text { 95\% } \\
\text { Confidence } \\
\text { interval }\end{array}$ & $p$-Value \\
\hline Omron & 74.4 & +3.2 & $(1.7-4.5)$ & $p<0.00$ I \\
iHealth BP5 & 75.1 & +3.9 & $(2.5-5.3)$ & $p<0.00$ I \\
iHealth BP7 & 77.9 & +6.7 & $(5.3-8.1)$ & $p<0.00$ I \\
Qardio & 74.1 & +2.9 & $(1.5-4.4)$ & $p<0.00$ I \\
Withings & 71.9 & +0.7 & $(-0.7-2.2)$ & $p=0.324$ \\
\hline
\end{tabular}

Table 3. Results of systolic blood pressure (BP) measurements of smartphone-compatible monitors, compared with the Withings Wireless Blood Pressure Monitor.

\begin{tabular}{lllll}
\hline & $\begin{array}{l}\text { Mean } \\
\text { systolic } \\
\text { BP }\end{array}$ & $\begin{array}{l}\text { Difference } \\
\text { with } \\
\text { Withings }\end{array}$ & $\begin{array}{l}\text { 95\% } \\
\text { Confidence } \\
\text { interval }\end{array}$ & $p$-Value \\
\hline iHealth BP5 & 124.0 & +1.9 & $(-0.9-4.5)$ & $p=0.193$ \\
iHealth BP7 & 126.0 & +3.9 & $(1.1-6.6)$ & $p=0.006$ \\
Qardio & 123.0 & +0.9 & $(-1.9-3.6)$ & $p=0.547$ \\
\hline
\end{tabular}

Table 4. Results of diastolic blood pressure (BP) measurements of smartphone-compatible monitors, compared with the Withings Wireless Blood Pressure Monitor.

\begin{tabular}{lllll}
\hline & $\begin{array}{l}\text { Mean } \\
\text { diastolic } \\
\text { BP }\end{array}$ & $\begin{array}{l}\text { Difference } \\
\text { with } \\
\text { Withings }\end{array}$ & $\begin{array}{l}95 \% \\
\text { Confidence } \\
\text { interval }\end{array}$ & $p$-Value \\
\hline iHealth BP5 & 124.0 & +3.2 & $(1.7-4.6)$ & $p<0.001$ \\
iHealth BP7 & 126.0 & +3.9 & $(4.5-7.4)$ & $p<0.001$ \\
Qardio & 123.0 & +0.9 & $(0.7-3.7)$ & $p=0.003$ \\
\hline
\end{tabular}

significant ( $p=0.193$ and $p=0.547$ respectively), while the difference with iHealth BP7 was significant $(p=0.006)$. These results are summarised in Table 3.

The average diastolic BP measured by Withings was 71.9. Differences with iHealth BP5, iHealth BP7 and Qardio were all statistically significant $(p<0.001$, $p<0.001$ and $p=0.003$ respectively). These results are summarised in Table 4.

\section{Measurement variability of the devices, systolic BP}

The statistical model assuming equal variances of the systolic BPs among devices was tested against the alternative model of unequal variances by comparing the respective -2 (log-likelihoods) within the appropriate linear mixed model. The difference of 10.603 was smaller than the critical value of 11.070 of a chi-square $($ d.f. $=5)$ distribution and therefore not significant.

\section{Measurement variability of the devices, diastolic BP}

For diastolic blood pressures the difference of 23.048 of the two -2 (log-likelihoods) was larger than the critical value of 11.070 of a chi-square (d.f. $=5$ ) distribution and therefore was significant.

\section{Discussion}

In the current study, inter-, and intra-device variability of four smartphone-compatible blood pressure monitors was compared with a handheld sphygmomanometer, a nonsmartphone-compatible oscillometric device and with each other. These monitors may be suitable for home monitoring, as their usage does not require assistance of trained healthcare staff and measurement results can be transferred automatically.

The results demonstrate that, compared with the conventional handheld sphygmomanometer and a nonsmartphone-compatible oscillometric device, compatible BP monitors tend to yield higher systolic and diastolic BPs. These differences were all statistically significant, except for the diastolic BP by the Withings Wireless Blood Pressure Monitor. However, these differences were also within the predefined range of $5 \mathrm{mmHg}$, except for the iHealth BP7. Intra-device variability was nonsignificant for systolic BP, but was significant for diastolic BP.

Validation studies of the devices used in the study have been published previously. ${ }^{10-13}$ These studies were all done in accordance with the European Society of Hypertension protocol, in which a device is compared with a mercury sphygmomanometer following a standardised protocol. ${ }^{16}$ These studies show a higher mean systolic and diastolic BP for the Omron M7, ${ }^{10}$ but a lower mean systolic and diastolic BP for the Withings Wireless Blood Pressure Monitor, ${ }^{13}$ the iHealth $\mathrm{BP} 5^{12}$ and the iHealth BP7. ${ }^{11}$ This is in contrast with the results of this study, in which these devices yielded higher mean systolic and diastolic BPs. This might be explained by the fact that in this study an overweight population was studied. Oscillometric devices tend to be more inaccurate in patients with a higher BMI. ${ }^{17}$

To our knowledge, studies comparing more than one oscillometric BP monitor with the gold standard have not been performed previously. Studies validating more than one oscillometric device do exist, ${ }^{13,18}$ however, all these studies describe several independently executed validation studies. These studies do not describe the validation of more than one oscillometric device in the same study population.

The fact that smartphone-compatible BP monitors tend to yield higher BPs, makes them less suitable for on-thespot measurements during outpatient clinic visits. However, the fact that these overestimates are within the $5 \mathrm{mmHg}$ range, and the intra-device consistency of the systolic BP measurements, makes them suitable for home monitoring. With daily measurements, more 
measurement results can be generated and the physician can adjust the therapeutic regimen on a trend instead on an on-the-spot measurement. It is hypothesised that these monitors give a reliable average systolic and diastolic BP based on daily measurements and the possibility of an incorrect therapeutic regimen change (defined as a physician changing the medication while the patient's BP has not changed) is small. It has already been shown that more frequent home monitoring of $\mathrm{BP}$, and changing therapeutic regimen when necessary, improves BP control. $^{6}$

There are, however, possibilities for further research. First, the results of this study need to be corroborated in a home situation, preferably with the patients using the devices themselves and without the assistance of trained healthcare staff. Second, the reliability of the BP monitors has to be investigated in the long-term. In a hospital setting, BP monitors are calibrated approximately every two years. ${ }^{19}$ However, as long-term comparison results are lacking, it remains unknown whether this calibration time-frame can be applied to oscillometric devices which are used in a home setting as well. ${ }^{19}$ Lastly, our study was conducted in adults with known cardiovascular disease. The results need to be corroborated in other populations, such as children and pregnant women.

Some limitations of our study design need to be mentioned. First of all, this study is meant to compare the four smartphone-compatible blood pressure measurements. It is not the primary purpose of this study to validate the devices. All devices have been validated previously. ${ }^{10-13}$ Therefore, the results should only be used for comparison purposes. Secondly, although the BP range of our population was quite wide, it need to be mentioned that measurements were done in a specific population. All patients were followed-up for myocardial infarction, and were therefore on antihypertensive drugs. Thirdly, no patients with (a history of) irregular heart rhythms were included in our study. All patients had regular sinus rhythm when they participated in the study. This means that the comparison made in our study cannot be extrapolated to patients who do have irregular heart rhythms. Lastly, our patients were tested in an outpatient clinic of a tertiary care centre. A researcher was present during all measurements. Although patients applied the devices themselves, patients were corrected in case of any mistakes in applying the devices.

\section{Conclusion}

In conclusion, we present a study comparing four validated smartphone-compatible BP monitors. Average inter-device variability was shown to be statistically significant, however, within the predefined range of $5 \mathrm{mmHg}$ for both systolic and diastolic BPs. Furthermore, devices showed comparable consistency for systolic BP, but less so for diastolic BP.

\section{Declaration of Conflicting Interests}

The authors declared no potential conflicts of interest with respect to the research, authorship, and/or publication of this article.

\section{Funding}

The authors received no financial support for the research, authorship, and/or publication of this article.

\section{References}

1. Perk J, De BG, Gohlke H, et al. European guidelines on cardiovascular disease prevention in clinical practice (version 2012). The Fifth Joint Task Force of the European Society of Cardiology and other societies on cardiovascular disease prevention in clinical practice (constituted by representatives of nine societies and by invited experts). Eur Heart $J$ 2012; 33: 1635-1701.

2. Pickering TG, Hall JE, Appel LJ, et al. Recommendations for blood pressure measurement in humans and experimental animals: Part 1: Blood pressure measurement in humans: A statement for professionals from the Subcommittee of Professional and Public Education of the American Heart Association Council on high blood pressure research. Circulation 2005; 111: 697-716.

3. Mancia G, Bertinieri G, Grassi G, et al. Effects of bloodpressure measurement by the doctor on patient's blood pressure and heart rate. Lancet 1983; 2: 695-698.

4. Arrieta A, Woods JR, Qiao N, et al. Cost-benefit analysis of home blood pressure monitoring in hypertension diagnosis and treatment: An insurer perspective. Hypertension 2014; 64: 891-896.

5. Mancia G, Fagard R, Narkiewicz K, et al. 2013 ESH/ESC guidelines for the management of arterial hypertension: The Task Force for the Management of Arterial Hypertension of the European Society of Hypertension (ESH) and of the European Society of Cardiology (ESC). Eur Heart J 2013; 34: 2159-2219.

6. Margolis KL, Asche SE, Bergdall AR, et al. Effect of home blood pressure telemonitoring and pharmacist management on blood pressure control: A cluster randomized clinical trial. JAMA 2013; 310: 46-56.

7. iHealth. iHealth wireless blood pressure monitor, https:// ihealthlabs.com/blood-pressure-monitors/wireless-bloodpressure-monitor/ (2016, accessed 9 May 2016).

8. Withings. Wireless blood pressure monitor, https://www. withings.com/eu/en/products/blood-pressure-monitor (2016, accessed 9 May 2016).

9. Qardio. QardioArm, https://www.getqardio.com/qardioarm-blood-pressure-monitor-iphone-android/ accessed 9 May 2016).

10. Coleman A, Steel S, Freeman P, et al. Validation of the Omron M7 (HEM-780-E) oscillometric blood pressure monitoring device according to the British Hypertension Society protocol. Blood Press Monit 2008; 13: 49-54.

11. Wang Q, Zhao H, Chen W, et al. Validation of the iHealth BP7 wrist blood pressure monitor, for self-measurement, according to the European Society of Hypertension International Protocol revision 2010. Blood Press Monit 2014; 19: 54-57.

12. Shang F, Zhu Y, Zhu Z, et al. Validation of the iHealth BP5 wireless upper arm blood pressure monitor for self-measurement according to the European Society of Hypertension 
International Protocol revision 2010. Blood Press Monit 2013; 18: 278-281.

13. Topouchian J, Agnoletti D, Blacher J, et al. Validation of four devices: Omron M6 Comfort, Omron HEM-7420, Withings BP-800, and Polygreen KP-7670 for home blood pressure measurement according to the European Society of Hypertension International Protocol. Vasc Health Risk Manag 2014; 10: 33-44.

14. Treskes RW, van der Velde ET, Eindhoven DC, et al. Comparison of four smartphone compatible blood pressure monitors. Comput Cardiol (2010) 2015; 42: 637-640.

15. Korotkoff N. On methods of studying blood pressure [in Russian]. Bull Imperial Mil Med Acad 1905; 11: 365-367.

16. O'Brien E, Pickering T, Asmar R, et al. Working Group on Blood Pressure Monitoring of the European Society of
Hypertension International Protocol for validation of blood pressure measuring devices in adults. Blood Press Monit 2002; 7: 3-17.

17. Maxwell MH, Waks AU, Schroth PC, et al. Error in bloodpressure measurement due to incorrect cuff size in obese patients. Lancet 1982; 2: 33-36.

18. Wong SN, Tz Sung RY and Leung LC. Validation of three oscillometric blood pressure devices against auscultatory mercury sphygmomanometer in children. Blood Press Monit 2006; 11: 281-291.

19. Bruining N, Caiani E, Chronaki C, et al. Acquisition and analysis of cardiovascular signals on smartphones: Potential, pitfalls and perspectives: By the Task Force of the e-Cardiology Working Group of European Society of Cardiology. Eur J Prev Cardiol 2014; 21: 4-13. 